\title{
A transparent stretchable sensor for distinguishable detection of touch and pressure by capacitive and piezoresistive signal transduction
}

\author{
Byeong-Ung Hwang ${ }^{1}$, Arsalan Zabeeb', Tran Quang Trung ${ }^{1}$, Long Wen ${ }^{1}$, Jae Deuk Lee', Young-In Choi', \\ Han-Byeol Lee', Ju Hyun Kim¹, Jeon Geon Han ${ }^{1}$ and Nae-Eung Lee $\mathbb{B}^{1,2,3}$
}

\begin{abstract}
Transparent stretchable (TS) sensors capable of detecting and distinguishing touch and pressure inputs are a promising development in wearable electronics. However, realization of such a device has been limited by difficulties in achieving optical transparency, stretchability, high sensitivity, stability, and distinguishable responsivity to two stimuli simultaneously. Herein, we report a TS sensor in which touch and pressure stimuli can be detected and distinguished on a substrate with a stress-relieving three-dimensional (3D) microstructured pattern providing multidirectional stretchability and increased pressure sensitivity. The TS capacitive device structure is a dielectric layer sandwiched between an upper piezoresistive electrode of a poly(3,4-ethylenedioxythiophene):poly(styrenesulfonate)/ionic liquid composite, which enables touch and pressure stimuli to be distinguished, and a lower electrode of metal/indium tin oxide/metal multilayer. The TS sensor array was demonstrated as a wearable input device for controlling a small vehicle. The TS touch-pressure sensor has great potential to be used as a multimodal input device for future wearable electronics.
\end{abstract}

\section{Introduction}

Over the past few years, numerous studies have been conducted to develop stretchable physical sensors for human-machine interfaces that can be worn or conformally attached to human skin ${ }^{1-24}$. Such body-attachable, stretchable physical sensors that are capable of detecting touch, pressure, strain, or temperature can also be used to monitor human activities ${ }^{2-18}$, health status ${ }^{18-21}$, and diseases $^{22-24}$. Integrating multiple sensors with different modalities as wearable input devices has been investigated as an approach to multimodal interactivity between humans and machines ${ }^{18,24-27}$. However, integrating multiple sensors

\footnotetext{
Correspondence: Nae-Eung Lee (nelee@skku.edu)

${ }^{1}$ School of Advanced Materials Science and Engineering, Sungkyunkwan University, Suwon, Gyeonggi-do 16419, Republic of Korea

${ }^{2}$ SKKU Advanced Institute of Nano Technology (SAINT), Sungkyunkwan

University, Suwon, Gyeonggi-do 16419, Republic of Korea

Full list of author information is available at the end of the article.
}

for multimodal interactivity often requires a large sensing area, complicated device structure, increased interconnection, and complicated fabrication processes ${ }^{18,24-27}$. One way of avoiding these limitations is to develop a sensor that can detect and distinguish two or more physical stimuli. For example, bimodal flexible sensors for simultaneous quantitative detection of temperature and pressure have been demonstrated ${ }^{28-31}$. However, reports of a single stretchable multimodal device and its array as a conformally wearable input device are rare.

Specifically, achieving such a wearable device for application to multimodal human-machine interfaces entails developing a stretchable sensor capable of distinguishing the inputs of touch and pressure (force). Since a wearable, stretchable touch-pressure sensor could be integrated into stretchable displays, optical transparency is also a desirable attribute. A few research groups have developed touch and pressure sensors with optical transparency or stretchability 
for the detection of touch and pressure stimuli ${ }^{32,33}$. In those projects, forming two or more sensors on one substrate was required for multimodal interactivity ${ }^{32}$. In addition, these sensors exhibited limitations in performance in terms of sensitivity and mechanical and optical characteristics when they were worn by humans or attached to human $\operatorname{skin}^{33}$. Therefore, it is of great interest to develop a transparent stretchable (TS) sensor for detection of touch and pressure stimuli that has mechanical stretchability, optical transparency, and high sensitivity, as well as the ability to detect and distinguish touch and pressure stimuli in a single device (see Table S1 in Supplementary Information).

This study presents a method of implementing a mutual capacitive-type bimodal touch and pressure sensor that meets the requirements described above. The substrate and each layer of the bimodal sensing device were fabricated using transparent materials. A three-dimensional (3D), strain-absorbing, micropatterned polydimethylsiloxane (PDMS) substrate with a bumpy morphology (curvilinearly connected bumps and valleys) ${ }^{34}$ was used to enhance the mechanical stability of the layers. An insulating transparent polyurethane (PU) layer was used as the dielectric. A transparent piezoresistive composite layer of poly(3,4ethylenedioxythiophene):poly(styrenesulfonate)/1-ethyl-3methylimidazolium tetracyanoborate (PEDOT:PSS/EMIMTCB) with good optical transparency $(\sim 84 \%$ at a thickness of $300 \mathrm{~nm}$ ) was used as the upper electrode, allowing distinguishable detection of touch and pressure stimuli. An ultrathin $\mathrm{Au} / \mathrm{ITO}$ (indium tin oxide)/Au (GIG) multilayer electrode with optical transparency $(\sim 65 \%)$ and a low sheet resistance $\left(\sim 11.5 \Omega \mathrm{sq}^{-1}\right)$ was used as the lower electrode. An excellent touch responsivity of $-23.4 \%$ and high pressure sensitivity of $0.1 \mathrm{kPa}^{-1}$ for the device with a PU dielectric layer thickness of $2.7 \mu \mathrm{m}$ were achieved. The 3D microstructure enhanced the pressure responsivity due to greater deformation of the piezoresistive upper electrode on the bumps. A $4 \times 4$ array sensor device with elasticity, transparency, and multifunctionality was then fabricated for demonstration of human-machine interfacing. The mechanical assessment of the single device and the array under stretching demonstrated that the sensitivity for touch-pressure stimuli was maintained after 10,000 stretching cycles at $30 \%$ stretching strain. Finally, it was successfully confirmed that a small vehicle could be controlled wirelessly by mounting the touch-pressure sensor array on a wrist. The TS touch-pressure sensor with a capacitive device structure has great potential as a wearable input device in future human-machine interfaces that require high functionality and multimodal interactivity.

\section{Materials and methods Materials}

A PDMS elastomer kit (Sylgard 184, Dow Corning), PU dispersion (PUD; 39-41 wt\% Alberdingk U3251,
Alberdingk Boley), PEDOT:PSS solution (1-1.3wt\%, CLEVIOS PH1000, Heraues), EMIM-TCB (1-ethyl-3methylimidazolium tetracyanoborate, Merck), and $\mathrm{Au}-\mathrm{Ni}$ woven conductive textile (Silverized Nylon/Spandex Knit SMP 130, Solueta Co. Ltd) were all used as purchased.

\section{Preparation of the PEDOT:PSS/EMIM-TCB composite}

PEDOT:PSS PH1000 was weighed, added to a vial, and stirred at high speed (>300 rpm), and the EMIM-TCB solution was added directly to the stirred PEDOT:PSS dispersion.

\section{Fabrication of the 3D micropatterned elastomeric substrate}

A mold with a 3D micropattern array on a glass substrate was fabricated by double photolithography using a positive photoresist ${ }^{34}$. Then, a 3D micropatterned PDMS was replicated from the mold, and a PU acrylate (PUA) master mold was replicated from the PDMS mold for repeated replication of the PDMS substrate. The micropatterned PDMS and PUA molds were repeatedly and interchangeably replicated.

\section{Finite element analyses}

3D FEA (ABAQUS software) enabled analysis of the full deformation mechanics and computation of mechanical responses under uniaxial loads. Four-node 3D solid elements and four-node shell elements were used for ITO (50 and $200 \mathrm{~nm}$ ) on the 3D micropatterned PDMS substrate and ITO $(200 \mathrm{~nm})$ on the planar PDMS substrate, respectively. The stress distribution of the ITO electrodes was estimated for a force load of $120 \mathrm{MPa}$ in the stretching mode.

\section{Fabrication and characterization of touch-pressure sensors}

The TS touch-pressure sensor was fabricated using the following procedure. To improve the adhesion of the electrode layers, a 3-nm thick $\mathrm{Al}_{2} \mathrm{O}_{3}$ layer was deposited via atomic layer deposition (ALD), followed by a 3-nm thick $\mathrm{Cr}$ layer that was deposited via $e$-beam evaporation, followed by deposition of GIG (Au-ITO-Au) layers using $e$-beam evaporation and advanced direct current (DC) sputter deposition ${ }^{35}$. PUDs with varying thicknesses were coated as the dielectric layer. Then, the PUD film was dried on a hot plate at $120^{\circ} \mathrm{C}$ for $2 \mathrm{~h}$. Next, a PEDOT:PSS/ EMIM-TCB composite piezoresistive material layer of $300 \mathrm{~nm}$ was spin coated as the pressure-sensing upper electrode material. The device was encapsulated by spincoated PDMS to prevent ambient interference during the measurement.

The sensor was measured by applying an alternating current (AC) pulse with an amplitude of $-3 \sim 3 \mathrm{~V}$ and frequency of $10 \mathrm{~Hz}$ to the upper electrode to induce dipole alignment inside the PU dielectric layer using a 
function generator (Tektronix AFG3102). The charges induced at the interface between the dielectric and the electrodes were detected as the momentary current at the lower electrode using a source measurement unit (HP 4145B).

\section{Demonstration of the $4 \times 4$ touch-pressure sensor array}

The output signal from the touch-pressure sensor array is electrical current, so we used a transimpedance amplifier (OPA2380, National Instruments, USA) to convert the electrical current signal (touch-pressure sensor array's output signal) into an electrical voltage signal. An inverting amplifier (LM324, National Instruments, USA) was used to amplify the converted voltage signal to meet the minimum required voltage level of a 10-bit analog to a digital converter (ADC), a resistor-capacitor (RC) low-pass filter with a cut-off frequency of $10 \mathrm{~Hz}$ was used to avoid higher frequencies, which are usually induced due to electrical power lines.

To demonstrate control of LED illumination, red and green LEDs were attached to two ports of the Arduino ${ }^{\oplus}$ UNO. In the absence of touch or pressure on the TS bimodal touch-pressure sensor array, the output current remained unchanged, as did the input of the microcontroller. When any sensor in the TS bimodal touchpressure sensor array was touched or pressure was applied, the output signal abruptly decreased. The Ardu-

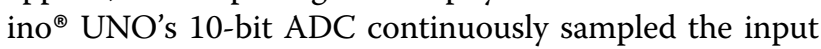
data and converted it to a digital signal for further processing. Based on the output data obtained from the sensor array, a voltage threshold was preset in the code written by Arduino ${ }^{\circledR}$ IDE (integrated development environment) to distinguish between touch and pressure stimuli. When a sensor was touched, the green LED started to blink with a delay of $500 \mathrm{~ms}$. Upon applying pressure to the sensor, the red LED blinked with the same delay time as the green LED. We demonstrated this in Movie S1, where real-time data for touch and pressure stimuli can be seen on an oscilloscope along with the blinking LEDs.

Furthermore, to demonstrate that our TS sensor array can be used for more complex human-machine interactivity, we used it to control a small toy car. In this demonstration, we affixed a $4 \times 4$ TS sensor array on a human forearm to control the direction and speed of a toy car. For data acquisition, we used the same analog circuitry as previously used for the LED demonstration, but for processing of the input signal, custom-made "master" and "slave" codes (terms widely used in embedded systems) written by Arduino ${ }^{\circledR}$ IDE and running in Arduino ${ }^{\circledR}$ UNO were used. Based on the processed data, appropriate commands were sent to the toy car using a Bluetooth low energy (BLE) module (HC-05, DigiKey, USA). Here, touching a sensor caused the toy car to move in a specific direction at a low speed, whereas applying pressure to the same device increased the vehicle speed in the same direction. Upon each detection of touch or pressure, the vehicle moved for $500 \mathrm{~ms}$ and then stopped if no subsequent touch or pressure stimulus was detected. By using three devices in a $4 \times 4$ sensor array, we successfully demonstrated movement of a toy car in the forward direction with low and high speeds, seamlessly turning left and right and then moving forward again at both low and high speed (Movie S2).

\section{Results \\ Concept of a transparent and stretchable touch-pressure sensor}

A schematic illustration of the TS touch-pressure sensor device mounted on the forearm of a human body is shown in Fig. 1a. The TS bimodal touch-pressure sensor can detect and distinguish between a light touch stimulus from a human finger and an intense pressure stimulus using changes in device capacitance and the resistance of the piezoresistive upper electrode, respectively. Figure $1 \mathrm{~b}$ is a schematic diagram of the structure of the mutual capacitive touch-pressure sensor. The sensor was fabricated by sequentially forming an ultrathin metal GIG lower electrode layer, a dielectric layer using a watersoluble dispersion of PU, a piezoresistive upper electrode layer using a PEDOT:PSS/EMIM-TCB composite, and an encapsulation layer of PDMS on a 3D micropatterned PDMS substrate ${ }^{34}$. The substrate design was inspired by a hill and valley geometry and has curvilinearly connected peaks and valleys without planar regions to minimize cracking of the layered materials in the upper part by avoiding an excessive stress concentration during stretching, thereby enhancing multidirectional lateral stretchability. Furthermore, increased deformation of the piezoresistive upper electrode on the bumps under vertical pressure leads to an increase in the sensitivity to pressure when the surface charges induced in the capacitor structure are read as an AC signal.

This single sensor has a diamond-shaped electrode design to reduce the overlap of the upper and lower electrodes (see Fig. 1c), improving both the optical transmittance and responsivity of the sensing area. The sensor responses to touch and pressure were measured by applying an $\mathrm{AC}$ square pulse voltage to the upper electrode to induce dipole alignment inside the PU dielectric layer, and then, the charges induced at the interface between the dielectric and the electrodes were detected as the momentary current by the lower electrode. The estimated response time for the detection of touch and pressure was approximately $0.14 \mathrm{~s}$. More detailed information on the fabrication process and measurements of the 3D micropatterned substrate and the touch-pressure sensor device are presented in the Materials and methods section. As shown in Fig. 1c, the optical transmittance of 

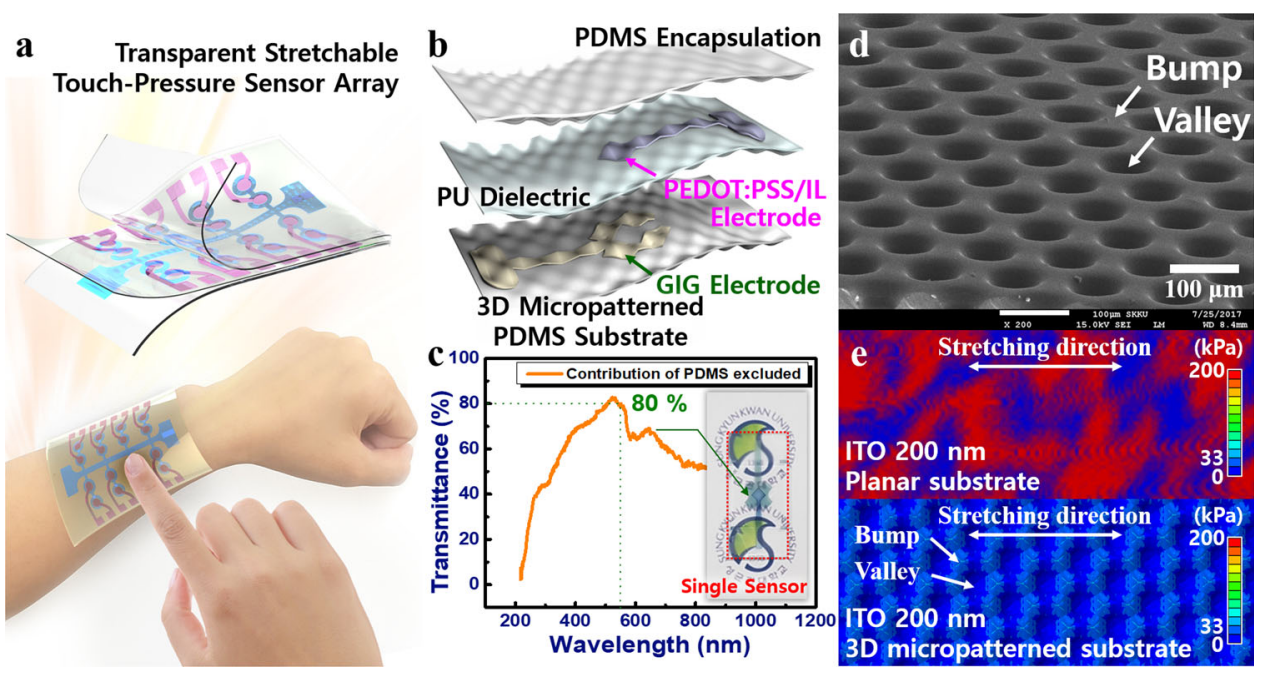

Fig. 1 Schematic diagram of the transparent and stretchable bimodal touch-pressure sensor. a Schematic illustration of the bimodal touchpressure sensor array. b Magnified view of the bimodal touch-pressure sensor, which comprises five stacked layers. c Optical transmittance spectra of the device region of the stacked electrodes and dielectric layer with the contribution of the PDMS substrate subtracted. Inset: photograph of the fabricated single sensor. $\mathbf{d}$ FE-SEM image of the 3D micropatterned PDMS substrate. e FEM simulation of the stress distribution of the ITO (200 nm) surface formed on planar (top) and 3D micropatterned (bottom) PDMS substrates under a stretching force of $120 \mathrm{MPa}$

the two stacked electrodes and dielectric layer (the inset in Fig. 1c), excluding the substrate, was approximately $80 \%$ at a wavelength of $550 \mathrm{~nm}$. The high optical transmittance is attributed to the design of the upper and lower electrodes with minimal vertical overlap. A top-view fieldemission scanning electron microscopy (FE-SEM) image of the 3D micropatterned PDMS substrate is shown in Fig. 1d. The efficient stress absorption capability of the 3D micropatterned PDMS substrate allowed development of the transparent and stretchable ITO-based lower electrode, in contrast to the high stress concentrations in the ITO layer on a planar PDMS substrate, which resulted in severe cracking (Fig. 1e).

\section{Transparent and stretchable electrodes on a 3D micropatterned elastomeric substrate}

To realize the TS bimodal touch-pressure sensor, it is critical to develop upper and lower electrodes with the electrical, optical, and mechanical properties required for the optical transparency, sensitivity, and mechanical stretchability of the device. The electrode layers were formed directly on a 3D micropatterned substrate using thin-film processes, and their electrical, optical, and mechanical properties were characterized (Fig. 2). For the TS lower electrode with low piezoresistivity and optical transparency, various structures with ultrathin metal layers of $\mathrm{Ti}$ (adhesion layer, $3 \mathrm{~nm}$ in thickness)/Au layers of varying thicknesses $(10,20$, and $30 \mathrm{~nm})$ and stacked GIG structures of $\mathrm{Au}(5 \mathrm{~nm}) / \mathrm{ITO} / \mathrm{Au}(5 \mathrm{~nm})$-Ti (adhesion layer, 3-nm thick) were fabricated using $e$-beam evaporation ( $\mathrm{Ti}$ and $\mathrm{Au}$ ) and an advanced $\mathrm{DC}$ sputter deposition (ITO) ${ }^{35}$ technique at $135^{\circ} \mathrm{C}$. A comparison of the sheet resistance and optical transmittance of the ultrathin $\mathrm{Au}$ electrode indicated that both decreased as the $\mathrm{Au}$ thickness increased (Fig. 2a). In the case of ultrathin $\mathrm{Au}$ layers only, optimization of the optical transmittance and sheet resistance was restricted. For example, the sheet resistance of the $\mathrm{Ti}(3 \mathrm{~nm}) / \mathrm{Au}(10 \mathrm{~nm})$ electrode with a total thickness of $13 \mathrm{~nm}$ was as high as $473 \Omega \mathrm{sq}^{-1}$, although it had a high optical transmittance of $70.8 \%$. When the Au thickness was increased to lower the sheet resistance, the optical transmittance increased significantly.

To overcome this difficulty, adequate levels of optical transmittance and sheet resistance could be achieved when a sandwiched structure of GIG with 50-200-nm thick ITO was used as a middle layer (Supplementary Fig. S1a). In addition to the sheet resistance and optical transmittance, we investigated the stability of the GIG lower electrode on the 3D micropatterned substrate under mechanical deformation. The data in Supplementary Fig. S1b, e, h, and Fig. $2 \mathrm{~b}$ show the measured resistance changes $\left(\Delta R / R_{0}\right.$, where $R_{0}$ is the initial resistance, and $\Delta R$ is the resistance increase under stretching) of the GIG structures under static stretching ranging from 0 to $30 \%$ elongation. The $\Delta R / R_{0}$ from static stretching decreased gradually with the increasing thickness of the ITO because the density of randomly generated cracks decreased (Supplementary Fig. S1d, g, j, and Fig. 2d). The GIG (200-nm ITO) electrode showed an increase in $\Delta R / R_{0}$ by a factor of $\sim 45$ at $30 \%$ strain after three cycles of static stretching (Fig. 2b). The effect of repetitive mechanical deformation on the GIG 

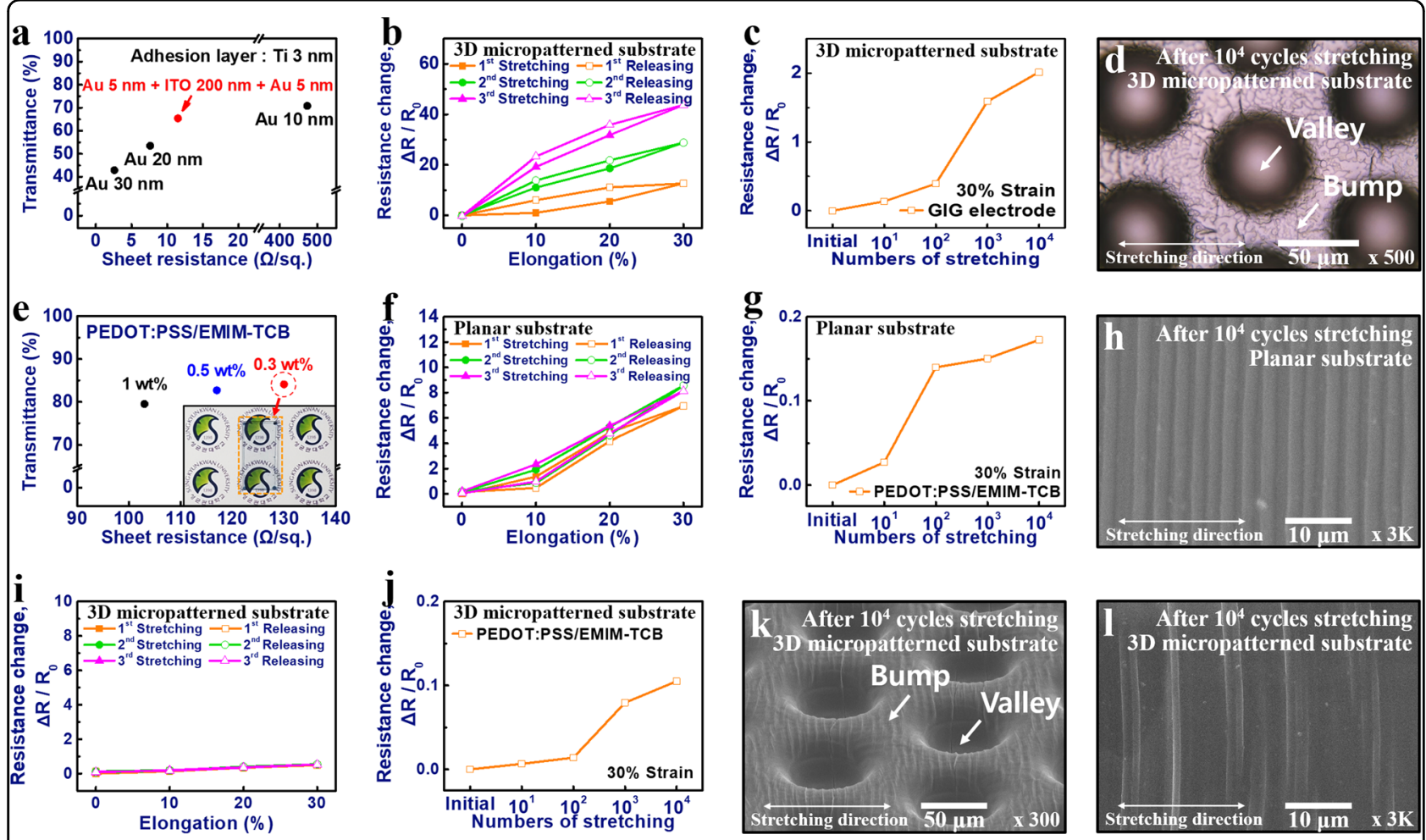

Fig. 2 Evaluation of transparent and stretchable electrodes. a Transmittance-sheet resistance graph of the different tested structures of the transparent lower electrode. b Resistance change $\left(\Delta R / R_{0}\right)$ versus elongation of the GIG [Au $(5 \mathrm{~nm}) / \mathrm{TO}(200 \mathrm{~nm}) / \mathrm{Au}(5 \mathrm{~nm})$-Ti (adhesion layer, $\left.\left.3 \mathrm{~nm}\right)\right]$ electrode on the 3D micropatterned substrate. c Resistance change $\left(\Delta R / R_{0}\right)$ in the GIG electrode $[\mathrm{Au}(5 \mathrm{~nm}) / \mathrm{TO}(200 \mathrm{~nm}) / \mathrm{Au}(5 \mathrm{~nm})-\mathrm{Ti}(\mathrm{adhesion}$ layer, $3 \mathrm{~nm})$ ] on the 3D micropatterned substrate as a function of stretching cycles at 30\% strain. $\mathbf{d}$ Surface optical image of the GIG electrode [Au (5 nm)/ ITO (200 nm)/Au (5 nm)-Ti (adhesion layer, $3 \mathrm{~nm}$ )] on the 3D micropatterned substrate after 10,000 stretching cycles, obtained using an optical microscope. e Optical transmittance of PEDOT:PSS/EMIM-TCB composite electrodes with varying concentrations of EMIM-TCB. Inset: photograph of the PEDOT:PSS/EMIM-TCB (0.3 wt\%) composite film on the planar PDMS substrate (orange-dashed line). f Resistance change ( $\triangle R / R_{0}$ ) versus elongation of the PEDOT:PSS/EMIM-TCB (0.3 wt\%) composite electrode on the planar PDMS substrate. $\mathbf{g}$ Resistance change $\left(\triangle R / R_{0}\right)$ in the PEDOT:PSS/EMIM-TCB $(0.3 \mathrm{wt} \%)$ composite electrode on the planar PDMS substrate as a function of stretching cycles at $30 \%$ strain. $\mathbf{h}$ Surface FE-SEM image of the PEDOT: PSS/EMIM-TCB (0.3 wt\%) composite electrode on planar PDMS substrate after 10,000 stretching cycles. i Resistance change $\left(\triangle R / R_{0}\right)$ versus elongation of the PEDOT:PSS/EMIM-TCB (0.3 wt\%) composite electrode on the 3D micropatterned substrate. $\mathbf{j}$ Resistance change $\left(\triangle R / R_{0}\right)$ in the PEDOT:PSS/EMIMTCB (0.3 wt\%) composite electrode on the 3D micropatterned substrate as a function of stretching cycles at 30\% strain. $\mathbf{k}$, I Surface FE-SEM images of the PEDOT:PSS/EMIM-TCB $(0.3 \mathrm{wt} \%)$ composite electrode on the 3D micropatterned substrate after 10,000 stretching cycles at $\mathbf{k} 300 \times$ and $\mathbf{I} 3,000 \times$

electrode stability was also investigated by a cyclic stretching test in which $\Delta R / R_{O}$ was measured after performing 10, 100, 1,000 and 10,000 stretching cycles with $30 \%$ strain (Fig. S1c, f, i, and Fig. 2c). The results indicated that the $\Delta R / R_{O}$ value increased by a factor of two after 10,000 stretching cycles (Fig. 2c). Although some degree of cracking occurred, the GIG structure maintained an effective current path, resulting in a small resistance change, indicating the possibility of using ITO as a TS electrode. As shown in Fig. 2a, a GIG (200-nm ITO) electrode with a sheet resistance of $11.5 \Omega \mathrm{sq}^{-1}$ and an optical transmittance of $\sim 65 \%$ at $550 \mathrm{~nm}$ was used for device fabrication.

To investigate the effect of cyclic stretching on the resistance change of the GIG (200-nm ITO) electrode, the surface morphological change after 10,000 stretching cycles at $30 \%$ strain was observed using an optical microscope. The optical image of the GIG electrode after 10,000 stretching cycles shown in Fig. 2d indicates that surface cracks were generated by aggressive cyclic stretching, and these cracks contributed to the observed $\Delta R / R_{0}$.

To understand the stress distribution on the ITO surface, finite-element modeling (FEM) simulations for the ITO layers (with thicknesses of 50 and $200 \mathrm{~nm}$ ) on the 3D micropatterned PDMS were carried out. The results on the thin ITO sample $(50 \mathrm{~nm})$ (Supplementary Fig. S2a) showed that the stress was focused on the peaks in a rhombus-like shape, leading to highly connected cracking of the ITO. Consequently, the loss of the current path can result in increased resistance, as experimentally observed (Supplementary Fig. S1d). The simulation results on the thicker ITO sample $(200 \mathrm{~nm})$ (Supplementary Fig. S2b), however, showed more random stress distribution on the 
peaks so that the cracks formed irregularly with higher connectivity, and the current paths were better maintained, which was consistent with the experimental results (Fig. 2d).

To elucidate the role of ultrathin Au layers in the GIG structure clearly, static and cyclic stretching tests and a surface morphology analysis were conducted in the same way for the 200-nm thick ITO layer only (sheet resistance of $31.7 \Omega \mathrm{sq}^{-1}$, optical transmittance of $73.9 \%$ ) on the 3D micropatterned substrate, with no stacking of ultrathin $\mathrm{Au}$ layers (Supplementary Fig. S3). The GIG multilayer electrode with ultrathin Au layers showed a lower sheet resistance and optical transmittance than the ITO layer alone. The data in Supplementary Fig. S3a indicate that the $\Delta R / R_{O}$ in the static stretching test increased up to a factor of 100 at $30 \%$ strain after three cycles of elongation from 0 to $30 \%$ strain. However, the $\Delta R / R_{O}$ value returned to the initial value. The cyclic test results after 10, 100, 1,000 , and 10,000 stretching cycles with $30 \%$ strain indicated that the $\Delta R / R_{O}$ value increased up to a factor of 15 for the ITO layer alone compared with increasing by a factor of two for the GIG structure (Supplementary Fig. $\mathrm{S} 3 \mathrm{~b})$. The results of the static and cyclic stretching tests both revealed that the resistance change was significantly lower in the GIG structure laminated with ultrathin $\mathrm{Au}$ layers than in the ITO layer alone. The image of the surface morphology after 10,000 stretching cycles showed that more cracks were generated on the ITO surface than on the GIG electrode surface (Supplementary Fig. S3c). Accordingly, when a mechanical strain is applied, the ultrathin metal layers may accommodate the stress to a certain degree, resulting in a reduction in cracking in the brittle ITO layer, which is expected to be very vulnerable to stress. In addition to the randomly connected ITO, even under cracked conditions, the ultrathin Au layers with a sandwich structure helped to form more effective current paths, which resulted in a low sheet resistance and less change in the electrical resistance due to cracking of the ITO layer.

To tune the compliance and piezoresistivity of the upper electrode, an ionic liquid with $0.3-1$ wt\% EMIMTCB was added to the flexible and transparent PEDOT: PSS layer, and their optical, electrical, and mechanical properties were evaluated (Fig. 2e-g and Supplementary Fig. S4). The EMIM-TCB in the PEDOT:PSS/EMIM-TCB composite contributed to the phase separation of PSS, leading to a structural change in the layer. In addition, it has been reported that as the concentration of EMIMTCB increases, the conductivity and stretchability improve, although the optical transmittance decreases slightly ${ }^{36-38}$. Therefore, we used spin-coatable PEDOT: PSS/EMIM-TCB composite materials for the stretchable and transparent piezoresistive upper electrode. The optical transmittance of the 300-nm thick PEDOT:PSS/
EMIM-TCB (0.3 wt\%) composite electrode formed on the planar PDMS substrate was $84 \%$ at a wavelength of $550 \mathrm{~nm}$ (Fig. 2e). The sheet resistance of this layer was $130 \Omega \mathrm{sq}^{-1}$. The $\Delta R / R_{O}$ data in Supplementary Fig. S4a and S4c indicate that $\Delta R / R_{O}$ under the static stretching condition increased by a factor of two at $30 \%$ strain after three repetitions of elongation from 0 to $30 \%$ strain, even though it returned to the initial value. However, the addition of lower concentrations of EMIM-TCB adversely affected the $\Delta R / R_{O}$ data after the static stretching tests (Fig. 2f). On the other hand, the cyclic test results after 10, $100,1,000$, and 10,000 stretching cycles with $30 \%$ strain indicated that the $\Delta R / R_{O}$ value increased by up to a factor of two for samples with 0.5 and $1 \mathrm{wt} \%$ EMIM-TCB (Supplementary Fig. S4b, d), compared with a factor of 0.17 for PEDOT:PSS with $0.3 \mathrm{wt} \%$ EMIM-TCB (Fig. 2g). These cyclic stretching test results revealed that the resistance change was significantly lower for PEDOT:PSS/ EMIM-TCB (0.3 wt\%) than for the samples with a higher concentration of EMIM-TCB (Fig. 2g). In addition, the solutions with > $1 \mathrm{wt} \%$ EMIM-TCB were too viscous to be solution processed and precipitated due to aggregation. As the concentration of EMIM-TCB mixed in PEDOT: PSS gradually increases, the viscosity increases and the tendency to form a gel becomes stronger ${ }^{38}$. In this case, the expansion and contraction characteristics are presumably improved, but the mechanical properties might be weakened when deformation repeatedly occurs. Therefore, the EMIM-TCB concentration was optimized at approximately $0.3 \mathrm{wt} \%$ to obtain better uniformity and mechanical stability in the upper electrode layer.

Surface morphological changes of the PEDOT:PSS/ EMIM-TCB (0.3 wt\%) composite layer on the planar PDMS after 10,000 stretching cycles at 30\% strain were investigated by FE-SEM (Fig. 2h). The FE-SEM images showed the formation of wrinkles on the surface in the direction of stretching. The wrinkles seem to have occurred during cyclic stretching, which may contribute to an improvement in the mechanical stability of the layer ${ }^{39,40}$. In Fig. $2 \mathrm{i}, \mathrm{j}$, the $\Delta R / R_{O}$ values of the PEDOT: PSS/EMIM-TCB (0.3 wt\%) composite layer on the 3D micropatterned substrate during the static and cyclic stretching tests are shown. The $\Delta R / R_{O}$ value increased only by a factor of 0.49 at $30 \%$ strain and did not show hysteresis in the resistance change. In addition, the $\Delta R / R_{O}$ value increased by a factor of only 0.1 after 10,000 stretching cycles at $30 \%$ strain. It turned out that there was a greater improvement in mechanical stability by laminating the TS composite electrode on a 3D micropatterned substrate than on a planar substrate. Thus, the PEDOT:PSS solution with $0.3 \mathrm{wt} \%$ EMIM-TCB was used to obtain high stretchability using a $3 \mathrm{D}$ micropatterned substrate while being highly responsive to pressure. The surface morphological change of the 
composite electrode on the 3D micropatterned substrate observed by FE-SEM after applying 10,000 stretching cycles also indicated the formation of wrinkles on the surface (Fig. 2k). The higher magnification FE-SEM image of a hill region of the substrate in Fig. 21 shows that fewer wrinkles were generated on the PEDOT:PSS/EMIM-TCB composite layer on the 3D micropatterned substrate in comparison with that on the planar PDMS substrate surface in Fig. 2h. Thus, the GIG and PEDOT:PSS/EMIMTCB $(0.3 \mathrm{wt} \%)$ composite electrodes were used as the lower and upper electrodes, respectively, for fabrication of the TS bimodal touch-pressure sensor device.

\section{Evaluation of the TS touch-pressure single sensor and array}

As a next step, this study attempted to improve the sensitivity of the proposed sensor for touch and pressure stimuli and evaluate its performance. The sensor responses to touch and pressure were measured by applying an $\mathrm{AC}$ pulse voltage of $-3 \sim 3 \mathrm{~V}$ at a frequency of $10 \mathrm{~Hz}$ to the upper electrode using a function generator, and the momentary current at the lower electrode was measured using a source measurement unit. It is known that the pressure range for touch is in the range of subtle pressure below $1 \mathrm{kPa}^{41}$. The pressure value during finger touch in our experiment was measured as approximately $0.5 \mathrm{kPa}$ using a force gauge. The experimental data in Supplementary Fig. S5 were obtained to identify and compare the characteristics of the TS bimodal touchpressure sensor on planar and 3D micropatterned substrates. Single devices were fabricated on planar (Supplementary Fig. S5a) and 3D micropatterned PDMS substrates (Supplementary Fig. S5b). The performances of these two sensors were compared. In this experiment, to clarify the mechanism of the sensor, an Au electrode was used as the lower electrode, and a piezoresistive PEDOT: PSS/PU composite was used as the upper electrode. The sensor on the planar PDMS substrate could not distinguish between touch and pressure stimuli. On the other hand, the sensor fabricated on the 3D micropatterned PDMS substrate showed an enhanced response to pressure stimulus, distinguishable from the response to touch stimulus. When the same pressure was applied to each device, the piezoresistive electrode on the peaks on the 3D micropatterned substrate was subject to more deformation than that on the planar surface, resulting in a larger resistance change and voltage drop in the upper electrode. Consequently, the current signal read from the lower electrode decreased, and the pressure could be effectively and distinguishably detected.

To measure and compare the sensitivities for touch and pressure stimuli according to the thickness of the PU dielectric layer, the current and capacitance changes of devices with upper electrodes of PEDOT:PSS/EMIM-TCB
$(0.3 \mathrm{wt} \%)$ and lower electrodes of $\mathrm{Au}$ on 3D micropatterned substrates were measured for PU layers with thicknesses of 6.8, 3.8, and $2.7 \mu \mathrm{m}$ (Fig. 3a-g). The current (Fig. $3 \mathrm{a}-\mathrm{c}$ ) and capacitance (Fig. $3 \mathrm{e}-\mathrm{g}$ ) data of the devices with varying $\mathrm{PU}$ layer thicknesses were measured for touch and pressure stimuli. The current responses of the device with a PU thickness of $2.7 \mu \mathrm{m}$ under pressures of 5 , 10, 15, and $20 \mathrm{kPa}$ are shown in Fig. 3h-k. The touch responsivity of the devices with different PU thicknesses and the pressure responsivity of the device with varying pressures are plotted as the current change $\left(\Delta I / I_{0}\right)$ in Fig. 3d, l, respectively. All the results in Fig. 3 were obtained under touch and finger pressure. Pressure during touch $(\sim 0.5 \mathrm{kPa})$ and pressure from a finger exerting force were monitored by a force gauge. The current response of the device with the $6.8-\mu \mathrm{m}$ thick PU layer for touch stimulus was as low as $-6.3 \%$ (Fig. 3a), and the current response of the devices with lower PU layer thicknesses of 3.8 and $2.7 \mu \mathrm{m}$ for touch stimulus increased to -14.0 and $-23.4 \%$, respectively, as shown in Fig. 3d. In general, the response to touch stimulus was enhanced with thinner PU dielectric layers. At PU thicknesses of 2.2 and $2.5 \mu \mathrm{m}$, however, there was no change in the response current, which is attributed to the high leakage of the thinner PU layers, corresponding to the level under normal operation of the sensor without touch. On the other hand, the capacitance changes $\left(\Delta C / C_{0}\right)$ of the devices with different PU layer thicknesses measured for two stimuli showed no significant difference in the responses to touch and pressure even though the $\Delta C / C_{0}$ response increased with decreasing PU layer thickness (Fig. $3 \mathrm{e}-\mathrm{g}$ ). To confirm the change in capacitance caused by finger pressure, pressures of $20 \mathrm{kPa}$ (Supplementary Fig. S6a), $40 \mathrm{kPa}$ (Supplementary Fig. S6b), and $80 \mathrm{kPa}$ (Supplementary Fig. S6c) were applied to the sensor with a PU layer thickness of $2.7 \mu \mathrm{m}$ fabricated on a 3D micropatterned substrate. The pressure was maintained using a force gauge. At pressures of 20 and $40 \mathrm{kPa}$, the capacitance change did not occur, while it significantly decreased at a high pressure of $80 \mathrm{kPa}$. The $\Delta C / C_{0}$ response did not change significantly in the low-pressure range, presumably because the top portion of the peak area in the pressurized zone, corresponding to only a small fraction of the total area, was deformed and, in turn, the capacitance change was negligible. Therefore, the sensor response to pressure was not induced by a change in the capacitance due to a reduction in the dielectric layer thickness, although at an elevated pressure of $80 \mathrm{kPa}$, the capacitance can partly contribute to a decrease in the response current.

The pressure sensitivity was quantitatively evaluated by applying varying force using a force machine. Pressures of 5-20 $\mathrm{kPa}$ were applied to the device for $1 \mathrm{~s}$, and the evaluation was performed as three measurements of the current for each force value. The current responses to 


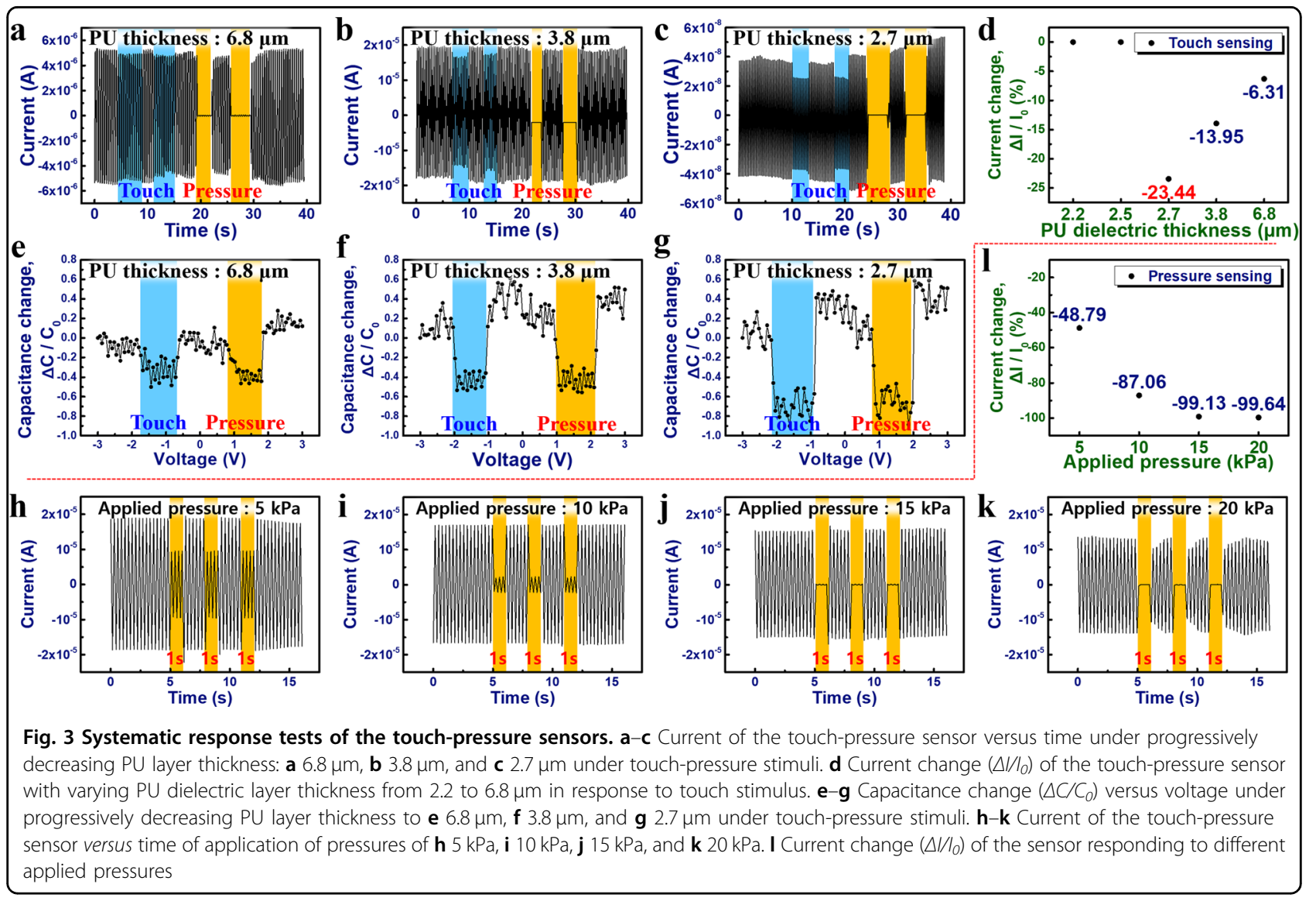

pressures of $5 \mathrm{kPa}$ (Fig. 3h), $10 \mathrm{kPa}$ (Fig. 3i), $15 \mathrm{kPa}$ (Fig. 3j), and $20 \mathrm{kPa}$ (Fig. 3k) indicated that the stronger the pressure was, the lower the current. These results are attributed to an increase in the resistance of the piezoresistive PEDOT:PSS/EMIM-TCB (0.3 wt\%) composite electrode due to the external pressure stimulus, which resulted in a voltage drop in the upper electrode followed by a decrease in current. To confirm the role of the top electrode in force sensing, a sensor using a piezoresistive PEDOT:PSS/EMIM-TCB composite layer only on the 3D micropatterned substrate was fabricated, and the resistance change at each pressure was measured. The data in Supplementary Fig. S7 indicate that the resistance change was negligible at a touch pressure of $0.5 \mathrm{kPa}$, while the change gradually increased with increasing pressure. Therefore, it was confirmed that the pressure-sensing capability during application of force can be ascribed to the change in the resistance of the upper electrode rather than the capacitance change. This principle enables the pressure stimulus to be distinguished from the touch stimulus. From the data in Fig. 3l, the pressure sensitivity was approximately $9.2 \% / \mathrm{kPa}$. At $20 \mathrm{kPa}$, the pressure responsivity was saturated at $-99.6 \%$. These results indicate that a pressure stimulus could not be detected and distinguished from touch via the capacitance change measurements but could be detected through current modulation due to the resistance change of the piezoresistive upper electrode under force.

The mechanical stability and durability of the sensor with an upper electrode of PEDOT:PSS/EMIM-TCB $(0.3 \mathrm{wt} \%)$ and a lower GIG (200-nm ITO) electrode were evaluated by measuring and comparing the responsivity under static stretching conditions in the stretching strain range of $0-30 \%$ elongation (Fig. 4a) and after cyclic stretching with $30 \%$ strain up to 10,000 stretching cycles (Fig. 4b). The measurement results before stretching are shown in Supplementary Fig. S8a. The detection results were obtained by applying 10,20 , and $30 \%$ elongation, and the results are presented in Supplementary Fig. S8b-d. Although the increase in strain was accompanied by a decrease in base current, the touch and pressure stimuli could be effectively sensed. Presumably, the decrease in the base current with stretching was caused by flattening the device structure during elongation, resulting in an increase in the resistance of the upper electrode. When the device was stretched up to $30 \%$ elongation and then returned to 20,10 , and $0 \%$, the base current was restored and both stimuli were well detected (Supplementary Fig. S8e-g). Figure 4a displays the sensitivity of the device to two stimuli under static stretching conditions. When 

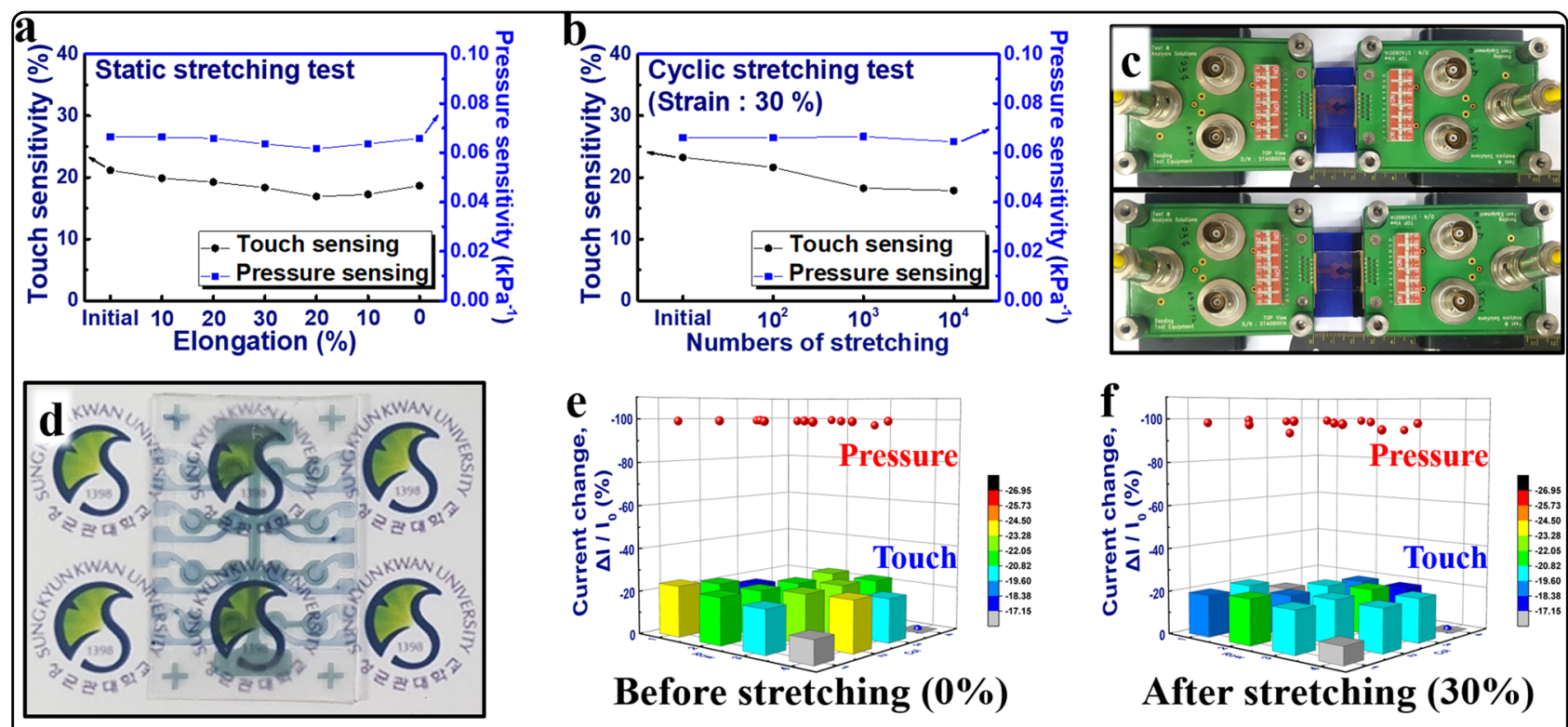

Fig. 4 Stretching tests of the transparent and stretchable single and $\mathbf{4} \times \mathbf{4}$ array touch-pressure sensor. a Sensitivity of a single sensor under static stretching from 0 to $30 \%$ with touch (black) and pressure (blue) stimuli. b Sensitivity of a single sensor as a function of stretching cycles at $30 \%$ with touch (black) and pressure (blue) stimuli. c Photographs of a single sensor with $0 \%$ (top) and $30 \%$ (bottom) stretching during the stretching test. d Photograph of the $4 \times 4$ sensor array. e, $\mathbf{f}$ Current change $\left(\Delta / / I_{0}\right)$ of the array under $\mathbf{e} 0 \%$ and $\mathbf{f} 30 \%$ strain with touch (bar) and pressure (scattered dot) stimuli

the stretching strain applied to the sensor increased from 0 to $30 \%$, the sensitivity for the two stimuli was stably maintained, which indicates the capability of detecting and distinguishing touch and pressure even under stretched conditions. Then, the mechanical stability was evaluated by applying 30\% stretching repeatedly and measuring the current responses under touch and pressure stimuli in sequence. The data in Supplementary Fig. S8h present the results obtained before cyclic stretching. The measurement data obtained after 100, 1,000, and 10,000 stretching cycles are shown in Supplementary Fig. S8i-k. Even after 10,000 stretching cycles, the base current was maintained to a certain degree, and the two stimuli could be distinguished and detected. The sensitivities of the device to touch and pressure after different stretching cycles (Fig. 4b) indicated that the sensor maintained its responsivity even after 10,000 stretching cycles at 30\% strain. The optical images in Fig. 4c show the transparent and stretchable touch-pressure sensor before and after stretching (30\%). When considering the similar strain-stress relations of the planar and 3D micropatterned substrates with upper or lower electrodes (Supplementary Fig. S9a) and a single device (Supplementary Fig. S9b) on them up to $30 \%$ strain, the 3D micropattern on the PDMS substrate enhances the mechanical stability of the electrodes and the device due to the efficient strain relief of the layers.

We also fabricated a $4 \times 4$ TS touch-pressure sensor array with a total area of $40 \times 60 \mathrm{~mm}^{2}$ and a single sensor size of $19.6 \mathrm{~mm}^{2}$ on a 3D micropatterned substrate using the TS electrodes described above (Fig. 4d). The results of the current responsivity $\left(\Delta I / I_{0}\right)$ to two stimuli applied on each sensor of the $4 \times 4$ array are illustrated in Fig. 4e. Most of the sensors in the array showed responsivity values close to $-20 \%$ and $-98 \%$ for touch and pressure, respectively. The evaluation results of each sensor in the array are shown in Supplementary Fig. S10. The $\Delta I / I_{O}$ responsivity of the $4 \times 4$ array was also measured by applying two stimuli to each sensor under 30\% stretching to evaluate their mechanical stability (Fig. 4f). In the stretched state, most of the sensors maintained responsivities comparable to those of the sensor without stretching. The evaluation results for each sensor under 30\% elongation are also shown in Supplementary Fig. S11. All the working sensors detected and distinguished touch and pressure stimuli. The results in Fig. 4 and Supplementary Figs. S8-S11 indicate the high stretchability, durability, and good responsivity of the TS bimodal sensor to touch and pressure stimuli.

\section{Demonstration of human-machine interaction using a touch-pressure sensor array}

We demonstrated that a TS touch-pressure sensor array $(4 \times 4)$ can be used for human-machine interfacing. Here, demonstrations of controlled illumination of light emitting diodes (LEDs) and movements of a small toy car were carried out. An analog circuit comprised of a 


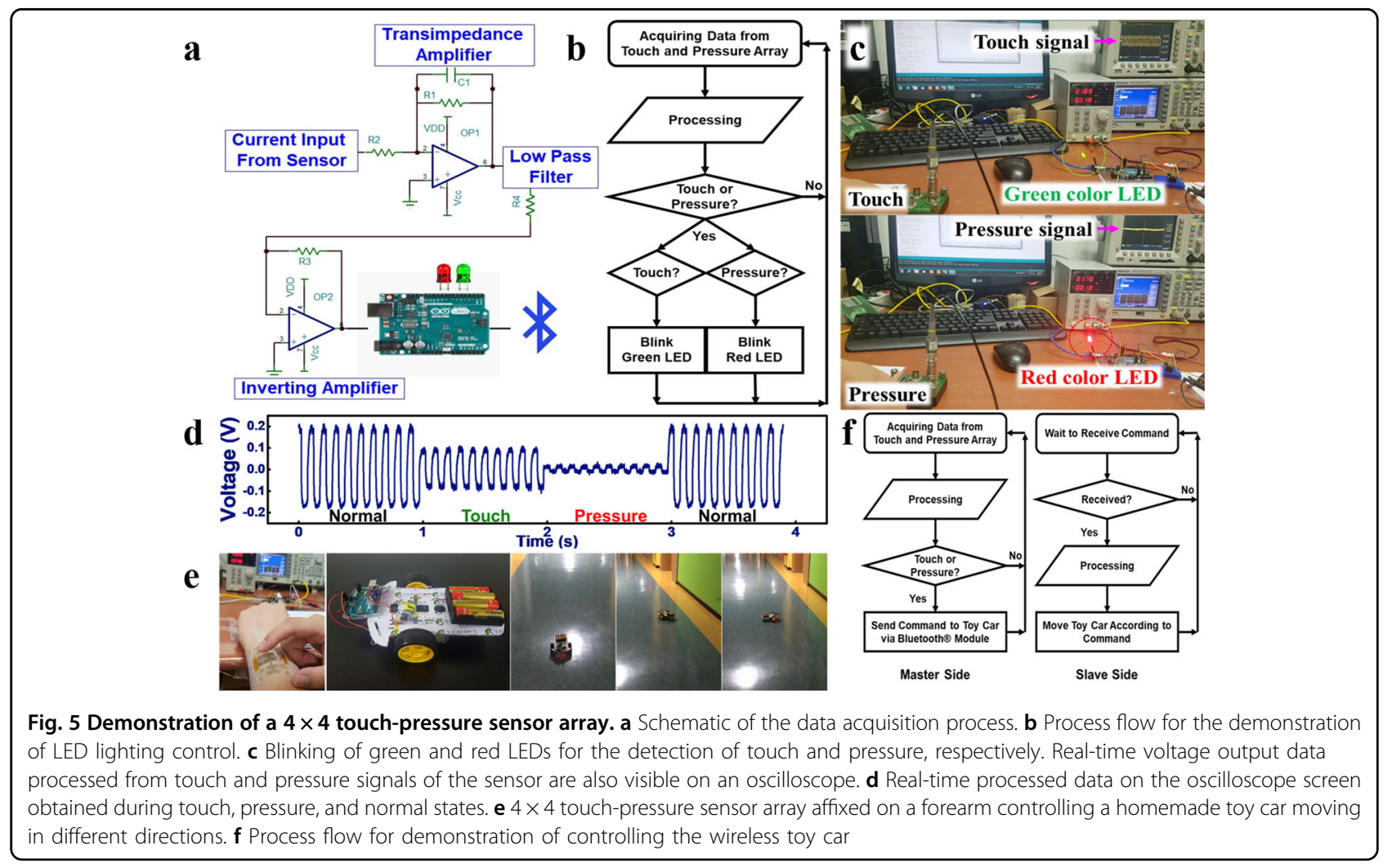

transimpedance amplifier and an operational amplifier was used to acquire and amplify the output signal of the sensor array, respectively. For data processing, custommade code written in Arduino IDE running in Arduino ${ }^{\circledR}$ UNO was used to distinguish between touch and pressure signals from the sensor. Figure $5 \mathrm{a}$ shows a schematic diagram for data acquisition from the touch-pressure sensor array. Figure $5 \mathrm{~b}$ shows a program flow for the first demonstration, which controls the lighting of green and red LEDs by touch and pressure stimuli, respectively, via two ports of the Arduino ${ }^{\circledast}$ UNO. Upon touching the sensor, a green LED blinks; upon pressurizing the sensor, a red LED blinks (Fig. 5c and Movie S1). Real-time data obtained by an oscilloscope applying stimuli are also plotted in Fig. 5d for a better understanding of the signal processing for touch and pressure stimuli. For the second demonstration, the array was mounted on a forearm and used to control a small toy car wirelessly using an $\mathrm{HC}-05$ Bluetooth ${ }^{\circledast}$ module. We used three devices from a $4 \times$ 4 sensor array to drive and control a homemade toy car wirelessly in three directions (forward, left, and right) at low and high speeds (Fig. 5e). For each of the three selected sensors, touching the sensor caused the car to move slowly in the specified direction, while applying pressure to the same sensor increased the vehicle speed in the same direction (Movie S2). For this second demonstration, the program flow of the "master side," which is the signal acquisition side, and the "slave side," which is the command execution side (toy car), is briefly explained in Fig. $5 f$.

\section{Discussion}

This study investigated a TS sensor capable of detecting and distinguishing touch and pressure stimuli. The multimodality of touch and pressure stimuli in a single sensing device can be an efficient alternative to conventional approaches in which two sensors with a single modality are integrated. Realizing force (pressure)-touch bimodality with the functionalities of stretchability and optical transparency in wearable input devices will require new approaches in material and device design, which have been impeded by challenges in developing materials with appropriate multifunctional properties and device structures for detecting distinguishable signals from a single device. Here, we propose a new approach using highly piezoresistive materials as an upper electrode in a capacitive-type structure so that applying pressure to the sensor modulates the resistivity and, in turn, the current induced between two electrodes in the capacitor. Thus, a touch stimulus is detected by measuring the current caused by the change in the capacitance, while a pressure stimulus is detected through the decrease in current caused by the voltage drop generated by the resistance increase in the upper electrode. This new design in materials, device structure, and signal reading methods allowed the detection and identification of both types of stimuli. 
The optical transparency and stretchability of the piezoresistive electrode for purposes of force detection were achieved by combining a compliant nanocomposite made of a conducting polymer, PEDOT:PSS/EMIM-TCB, with 3D geometric engineering on a 3D micropatterned PDMS substrate. The formation of a compliant, conducting polymer composite on a 3D micropatterned PDMS substrate also made it possible to obtain high responsivity to pressure due to the larger deformation on peaks under vertical pressure. One more challenge was to fabricate a TS electrode with high mechanical stretchability, optical transparency, and electrical conductance. Although conventional ITO materials have been tested for use as stretchable electrodes, cyclic stretching causes severe cracking of ITO thin films, and their mechanical and electrical stabilities are very poor ${ }^{42-44}$. The GIG electrode based on transparent, conductive but brittle ITO thin films sandwiched between ultrathin metal layers formed on a stress-relieving 3D micropatterned elastomeric substrate by conventional thin-film processing shows potential for developing TS electrodes for many applications, although further improvements in optical transparency are necessary. It was confirmed that the use of a 3D micropatterned substrate not only improved the responsivity to pressure but also increased the mechanical stability of the device under stretching.

The development of a transparent and stretchable touch-pressure sensor via new methods in electrode materials, substrate, and device structuring could be applied to the fabrication of a sensor array. This sensor array could be mounted on the skin, and the possibility of multiple input functions was successfully demonstrated. Since the proposed bimodal sensor could receive inputs of various information types in a limited space, both the high functionality and multifunctionality of the sensor will be advantageous for a miniaturized form factor. In addition, the sensor can be applied to a wearable input system for which the input of 3D information is possible, whereas existing touch sensors recognize only twodimensional (2D) information ${ }^{13,45}$. Despite the results showed in our work, however, the electrical and mechanical stability of the two electrodes need to be improved further through optimization of material design and geometric engineering to minimize the strain caused by external stresses associated with the bodily motions of wearers.

In conclusion, we developed a new kind of transparent and stretchable mutual capacitive-type touch-pressure sensor based on a transparent and stretchable PEDOT: PSS/EMIM-TCB composite piezoresistive upper electrode and GIG multilayer lower electrode. The transparent and stretchable piezoresistive electrode enables the sensor to detect and distinguish touch and pressure.
Combining the highly effective stress absorption ability of the 3D micropatterned substrate with the enhanced deformation of the piezoresistive electrode near peaks under vertical pressure provides high responsivity to touch and pressure stimuli and mechanical stability. This study demonstrates that the proposed sensor could be easily integrated into an ergonomic wearable device as an input unit, and its transparency and stretchability would enable it to be used as a patch-type sensor mounted on the human body. Moreover, when the sensor is integrated with other components, such as signal processing and data transmitting units, higher levels of interactivity could be demonstrated, which implies that the TS sensor has great potential as a key element of future human-machine interfaces. Furthermore, the touchpressure bimodality of the sensor can be integrated into a stretchable touch panel for stretchable display technology. In this respect, the proposed sensor has great potential for future wearable electronics.

\section{Acknowledgements}

This research was supported by the Samsung Display Corp. This work was also supported by the Basic Science Research Program (2016R1A2A1A05005423) through the National Research Foundation (NRF), funded by the Ministry of Science, ICT \& Future Planning.

\section{Author details}

${ }^{1}$ School of Advanced Materials Science and Engineering, Sungkyunkwan University, Suwon, Gyeonggi-do 16419, Republic of Korea. ${ }^{2}$ SKKU Advanced Institute of Nano Technology (SAINT), Sungkyunkwan University, Suwon, Gyeonggi-do 16419, Republic of Korea. ${ }^{3}$ Samsung Advanced Institute for Health Sciences and Technology (SAIHST), Sungkyunkwan University, Suwon, Gyeonggi-do 16419, Republic of Korea

\section{Conflict of interest}

The authors declare that they have no conflict of interest.

\section{Publisher's note}

Springer Nature remains neutral with regard to jurisdictional claims in published maps and institutional affiliations.

Supplementary information is available for this paper at https://doi.org/ 10.1038/s41427-019-0126-X.

Received: 30 November 2018 Revised: 8 March 2019 Accepted: 25 March 2019.

Published online: 24 May 2019

\footnotetext{
References

1. Trung, T. Q. et al. An omnidirectionally stretchable photodetector based on organic-inorganic heterojunctions. ACS Appl. Mater. Interfaces 9, 35958-35967 (2017).

2. Wang, X. et al. A highly stretchable transparent self-powered triboelectric tactile sensor with metallized nanofibers for wearable electronics. Adv. Mater. 30, 1706738 (2018).

3. Kang, M. et al. Graphene-based three-dimensional capacitive touch sensor for wearable electronics. ACS Nano 11, 7950-7957 (2017).

4. Roh, E., Hwang, B.-U., Kim, D., Kim, B.-Y. \& Lee, N.E. Stretchable, transparent, ultrasensitive, and patchable strain sensor for human-machine interfaces
} 
comprising a nanohybrid of carbon nanotubes and conductive elastomers. ACS Nano 9, 6252-6261 (2015)

5. Hwang, B.-U. et al. Transparent stretchable self-powered patchable sensor platform with ultrasensitive recognition of human activities. ACS Nano $\mathbf{9}$, 8801-8810 (2015)

6. Lee, J. et al. A stretchable strain sensor based on a metal nanoparticle thin film for human motion detection. Nanoscale 6, 11932-11939 (2014).

7. Shi, J. et al. Graphene reinforced carbon nanotube networks for wearable strain sensors. Adv. Funct. Mater. 26, 2078-2084 (2016).

8. Wang, C. et al. Carbonized silk fabric for ultrastretchable, highly sensitive, and wearable strain sensors. Adv. Mater. 28, 6640-6648 (2016).

9. Park, J. J., Hyun, W. J., Mun, S. C., Park, Y. T. \& Park, O. O. Highly stretchable and wearable graphene strain sensors with controllable sensitivity for human motion monitoring. ACS Appl. Mater. Interfaces 7, 6317-6324 (2015).

10. Ge, J. et al. A stretchable electronic fabric artificial skin with pressure-, lateral strain-, and flexion-sensitive properties. Adv. Mater. 28, 722-728 (2016).

11. Kim, K. K. et al. Highly sensitive and stretchable multidimensional strain sensor with prestrained anisotropic metal nanowire percolation networks. Nano Lett. 15, 5240-5247 (2015).

12. Choi, D. Y. et al. Highly stretchable, hysteresis-free ionic liquid-based strain sensor for precise human motion monitoring. ACS Appl. Mater. Interfaces $\mathbf{9}$, 1770-1780 (2017)

13. Kim, C.-C., Lee, H.-H., Oh, K. H. \& Sun, J.-Y. Highly stretchable, transparent ionic touch panel. Science 12, 682-687 (2016).

14. Jung, $\mathrm{S}$. et al. Reverse-micelle-induced porous pressure-sensitive rubber for wearable human-machine interfaces. Adv. Mater. 26, 4825-4830 (2014).

15. Kim, N., Lim, T., Song, K, Yang, S. \& Lee, J. Stretchable multichannel electromyography sensor array covering large area for controlling home electronics with distinguishable signals from multiple muscles. ACS Appl. Mater. Interfaces 8, 21070-21076 (2016)

16. Yao, S. \& Zhu, Y. Wearable multifunctional sensors using printed stretchable conductors made of silver nanowires. Nanoscale 6, 2345-2352 (2014).

17. Park, J. et al. Tactile-direction-sensitive and stretchable electronic skins based on human-skin-inspired interlocked microstructures. ACS Nano 8 , 12020-12029 (2014)

18. Trung, T. Q., Ramasundaram, S., Hwang, B.-U. \& Lee, N.-E. An all-elastomeric transparent and stretchable temperature sensor for body-attachable wearable electronics. Adv. Mater. 28, 502-509 (2016).

19. Roh, E., Lee, H.B., Kim, D.-I. \& Lee, N.-E. A solution-processable, omnidirectionally stretchable, and high-pressure-sensitive piezoresistive device. Adv. Mater. 29, 1703004 (2017).

20. Park, H. et al. Stretchable array of highly sensitive pressure sensors consisting of polyaniline nanofibers and au-coated polydimethylsiloxane micropillars. ACS Nano 9, 9974-9985 (2015).

21. Amjadi, M., Turan, M., Clementson, C. P. \& Sitti, M. Parallel microcracks-based ultrasensitive and highly stretchable strain sensors. ACS Appl. Mater. Interfaces 8, 5618-5626 (2016).

22. Park, Y. et al. Microtopography-guided conductive patterns of liquid-driven graphene nanoplatelet networks for stretchable and skin-conformal sensor array. Adv. Mater. 29, 1606453 (2017).

23. You, I. et al. Stretchable e-skin apexcardiogram sensor. Adv. Mater. 28 6359-6364 (2016).

24. Son, D. et al. Multifunctional wearable devices for diagnosis and therapy of movement disorders. Nat. Nanotechnol. 9, 397-404 (2014).
25. $\mathrm{Xu}$, B. et al. An epidermal stimulation and sensing platform for sensorimotor prosthetic control, management of lower back exertion, and electrical muscle activation. Adv. Mater. 28, 4462-4471 (2016).

26. Yamamoto, Y. et al. Printed multifunctional flexible device with an integrated motion sensor for health care monitoring. Sci. Adv. 2, e1601473 (2016).

27. Zhao, S. \& Zhu, R. Electronic skin with multifunction sensors based on thermosensation. Adv. Mater. 29, 1606151 (2017).

28. Tien, N. T. et al. A flexible bimodal sensor array for simultaneous sensing of pressure and temperature. Adv. Mater. 26, 796-804 (2014).

29. Kim, D.-I. et al. A sensor array using multi-functional field-effect transistors with ultrahigh sensitivity and precision for bio-monitoring. Sci. Rep. 5, 12705 (2015).

30. Park, J., Kim, M., Lee, Y., Lee, H. S. \& Ko, H. Fingertip skin-inspired microstructured ferroelectric skins discriminate static/dynamic pressure and temperature stimuli. Sci. Adv. 1, e1500661 (2015)

31. Bae, G. Y. et al. Pressure/temperature sensing bimodal electronic skin with stimulus discriminability and linear sensitivity. Adv. Mater. 30, 1803388 (2018).

32. Song, J.-K. et al. Wearable force touch sensor array using a flexible and transparent electrode. Adv. Funct. Mater. 27, 1605286 (2017).

33. Huang, Y. et al. A flexible touch-pressure sensor array with wireless transmission system for robotic skin. Rev. Sci. Instrum. 87, 065007 (2016).

34. Lee, H.-B. et al. Mogul-patterned elastomeric substrate for stretchable electronics. Adv. Mater. 28, 3069-3077 (2016).

35. Wen, L., Sahu, B. B. \& Han, J. G. Development and utility of a new 3-D magnetron source for high rate deposition of highly conductive ITO thin films near room temperature. Phys. Chem. Chem. Phys. 20, 4818-4830 (2018).

36. Badre, C, Marquant, L. Alsayed, A. M. \& Hough, L. A. Highly conductive poly (3,4-ethylenedioxythiophene):poly(styrenesulfonate) films using 1-ethyl-3methylimidazolium tetracyanoborate ionic liquid. Adv. Funct. Mater. 22, 2723-2727 (2012)

37. Kee, S. et al. Controlling molecular ordering in aqueous conducting polymers using ionic liquids. Adv. Mater. 28, 8625-8631 (2016).

38. Teo, M. Y. et al. Highly stretchable and highly conductive PEDOT:PSS/ionic liquid composite transparent electrodes for solution-processed stretchable electronics. ACS Appl. Mater. Interfaces 9, 819-826 (2017).

39. Xu, F. \& Zhu, Y. Highly conductive and stretchable silver nanowire conductors. Adv. Mater. 24, 5117-5122 (2012).

40. Lee, G.-B., Sathi, S. G., Kim, D.-Y., Jeong, K.-U. \& Nah, C. Wrinkled elastomers for the highly stretchable electrodes with excellent fatigue resistances. Polym. Test. 53, 329-337 (2016).

41. Zang, Y., Zhang, F., Di, C.-a \& Zhu, D. Advances of flexible pressure sensors toward artificial intelligence and health care applications. Mater. Horiz. 2, 140-156 (2015)

42. Yang, S., Su, B., Bitar, G. \& Lu, N. Stretchability of indium tin oxide (TTO) serpentine thin films supported by Kapton substrates. Int. J. Fract. 190, 99-110 (2014).

43. Gutruf, P. et al. Transparent functional oxide stretchable electronics: microtectonics enabled high strain electrodes. NPG Asia Mater. 5, e62 (2013).

44. Lien, S.-Y., Nautiyal, A. \& Lee, S. J. Optoelectronic properties of indium-tin oxide films deposited on flexible and transparent poly(dimethylsiloxane) substrate. Jpn. J. Appl. Phys. 52, 115801 (2013).

45. Choi, T. Y. et al. Stretchable, transparent, and stretch-unresponsive capacitive touch sensor array with selectively patterned silver nanowires/reduced graphene oxide electrodes. ACS Appl. Mater. Interfaces 9, 18022-18030 (2017) 